\title{
Carnets
}

Revue électronique d'études françaises de l'APEF

Deuxième série - 16| 2019

Le Récit inachevé: études sur Mai 68

\section{Romain Gary et Mai 68. Mots d'ordre et de désordre}

Jacques Isolery

\section{(2) OpenEdition}

\section{Journals}

\section{Édition électronique}

URL : http://journals.openedition.org/carnets/9751

DOI : 10.4000/carnets.9751

ISSN : 1646-7698

Éditeur

APEF

\section{Référence électronique}

Jacques Isolery, «Romain Gary et Mai 68. Mots d'ordre et de désordre », Carnets [En ligne], Deuxième série - 16 | 2019, mis en ligne le 31 mai 2019, consulté le 10 novembre 2019. URL : http:// journals.openedition.org/carnets/9751; DOI : 10.4000/carnets.9751

Ce document a été généré automatiquement le 10 novembre 2019.

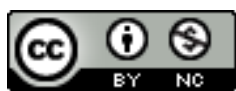

Carnets est mis à disposition selon les termes de la licence Creative Commons - Atribution - Pas d'utilisation commerciale 4.0 International. 


\title{
Romain Gary et Mai 68. Mots d'ordre et de désordre
}

\author{
Jacques Isolery
}

Pour diverses raisons, il est vrai que «Romain Gary n'a jamais été reconnu comme intellectuel, ni de son vivant, ni depuis sa mort.» (Lara, 2014: 129) Pourtant, une grande partie de ses œuvres, de ses entretiens ou écrits à caractère autobiographique tourne autour de problèmes qui, sans être politiques au sein restreint du terme, n'en participent pas moins à des débats de société, à des inquiétudes éthiques ou esthétiques. Romain Gary fera dire à l'ambassadeur Danthès, dans Europa : «Je ne crois pas qu'il y ait une éthique digne de l'homme qui soit autre chose qu'une esthétique assumée dans la vie jusqu'au sacrifice de la vie elle-même... » (Gary, 1972 : 177). Il existe un malentendu qui porte sur le hiatus entre son gaullisme, la critique de la société occidentale moderne, la revendication de son appartenance à une pensée de gauche et le regard souvent ironique qu'il a pu porter sur Mai 68. Parmi les préoccupations qui ont agité cette période mais dont aucune n'est aujourd'hui véritablement résolue, Gary s'est profondément interrogé sur la liberté sexuelle, la drogue, la surpopulation, l'urbanisme délirant, l'écologie, le statut de la femme, le pacifisme, le racisme... Tous ces mots d'ordre et de désordre d'un homme d'ordre et de désordre renvoient à des données à la fois collectives et individuelles, locales et globales, mais aussi à des expériences dont Gary redoutait qu'elles ne fassent progressivement de l'être humain une simple «barbaque » à disposition de toutes les formes possibles de totalitarisme, $\mathrm{y}$ compris celui d'un libéralisme sauvage et destructeur de toutes valeurs éthiques. À travers une exploration des romans de cette époque (mais pas seulement), des interviews et autres confidences, ainsi que du comportement de Gary vis à vis de tous les mouvements collectifs, on essayera de jauger la pertinence (voire l'impertinence) de ses positions à l'égard de quelques grands thèmes soixante-huitards, de sonder la valeur prophétique de celui qu'on considérait à l'époque comme un gaulliste ringard ou " comme une sorte de hippie gaulliste, démodé » (Anissimov, 2006: 606). Aujourd'hui, sa conception de la littérature, de l'art et de la société en général n'a, semble-t-il, rien perdu de son actualité mais bien au contraire gagné en acuité visionnaire, conservé son 
ironie décapante et sa truculence picaresque. Pas plus que n'a perdu en pertinence sa position ambiguë, décalée, à l'égard de l'idéalisme humaniste.

\section{Il y a en France, 38000 communes... nous en sommes à la seconde (Sciences-Po)}

2 Il faut bien avouer que Mai 68 ne semble pas, à première vue, avoir beaucoup marqué la vie ou l'œuvre de l'écrivain. À cette époque, il est «chargé de mission depuis un an auprès du ministre de l'Information, Georges Gorse » (Bona, 1987: 308) et se bat en France contre la censure (Gary, 1970b) dont il est lui-même victime à propos de Les Oiseaux vont mourir au Pérou, un film tourné avec Jean Seberg, à partir de la nouvelle du même titre ${ }^{1}$. Pas plus que de Gaulle au moment d'adresser ses vœux en janvier 1968 aux Français, et ce malgré les grandes grèves de 67, Romain Gary n'a anticipé les événements qui allaient mettre le feu aux poudres non seulement universitaires ${ }^{2}$ mais aux usines et à bien d'autres secteurs de la vie économique et sociale jusqu'à paralyser le pays. Eut-il fréquenté l'université, peut-être aurait-il éprouvé cruellement lui aussi «la médiocrité autoritaire des enseignants, leur enseignement soporifique ([...]), l'arrogance de l'administration universitaire et de ses sbires, l'infantilisation des étudiants, l'arbitraire, la hiérarchie, la contrainte et le contrôle omniprésents " (Guégan et Narodetzki, 1998 : 11). Eut-il été plus jeune, peut-être aurait-il ressenti cet ennui généralisé, dû entre autres à l'impossibilité de satisfaire au grand jour les nouvelles aspirations, sexuelles en particulier, de la jeunesse.

3 Mais en 68, Gary n'appartient plus depuis longtemps à cette jeunesse... Une fois de plus, il traverse une période difficile de sa vie. Il est en train de se séparer de Jean Seberg, mais pour la voir et la protéger il se rend très souvent aux États-Unis où l'actualité politique se manifeste à travers les problèmes de violence et de racisme auxquels sont confrontés les Américains en général et Jean en particulier: les revendications des Black Panters, les assassinats successifs de Jack Kennedy en 1963, celui de Bob Kennedy le 6 juin 1968, de Martin Luther King le 4 avril, la guerre froide, Cuba, le Viêt-Nam, etc. À Paris, les revendications idéalistes des étudiants, celles plus pragmatiques des ouvriers, les manifestations de rue, les récupérations politiques, tout cela exprime certes un profond malaise sociétal mais ne revêt pas, du moins à ses yeux, un tel caractère dramatique : "Comparée aux drames Biafra, du Vietnam, de l'intervention soviétique à Prague, il trouve que "la Révolution des étudiants de Paris ressemblerait singulièrement à une émeute de souris dans un fromage” " (Savatier, 2008). D’ailleurs, Gary ne voit dans ce qu'on a falsifié sous le terme unificateur de « Mai 68 » que la face estudiantine, parisienne et finalement très ponctuelle d'un phénomène qui, selon JeanClaude Michéa, n'a en réalité globalement pris fin qu'en 1974, au moment

du coup d'État libéral organisé au Chili - en septembre 1973 - par le général Pinochet et ses commanditaires états-uniens [...] la «révolution" n'était donc pas uniquement une fête immense et joyeuse (une Rebel-Pride ensoleillée sur fond de musique woodstockienne) : elle pouvait même conduire ceux qui la prenaient au sérieux à devoir mourir sous la torture [...] C'est à ce moment précis que les lampions ont commencé à s'éteindre. (Michéa, 2008 : 125)

Ce qui révolte principalement Gary, c'est la répression des manifestations de cette jeunesse libertaire ou militante, qui le pousse à démissionner de son poste et à refuser de « rallier les "répresseurs" » (Bona, 1987 : 316). Car, depuis toujours, «Gary éprouve 
une sympathie spontanée pour les victimes » (Todorov, $2002: 102)$. En revanche, il n'en a jamais voulu endosser le costume, pas plus que celui du héros. Il considère que «l'expérience de victime de vous accorde aucun privilège durable» (Todorov, 2002 : 103), et certainement pas celui d'inverser les rôles : «C'est tout de même triste lorsque les Juifs se mettent à rêver d'une Gestapo juive et les Noirs d'un Ku-Klux-Klan noir... » (Gary, 1970: 218). Cette tristesse, c'est celle qui accompagne la conscience de l'inhumanité fondamentale de l'humain et entrevoit l'« égalité dans la chiennerie » (Gary, 1970 : 218). Gary est incapable de s'identifier à quelque groupe que ce soit, hormis peut-être au vieux souvenir des "Français libres". Or,

Pendant que Sartre s'amuse à vendre La Cause du peuple devant les usines Renault de Billancourt, tu dénonces dans La Tête coupable, la révolution culturelle en Chine. Les Racines du ciel, Les Mangeurs d'étoiles, Chien blanc parlent avec une lucidité impitoyable de ce que font les opprimés et les humiliés quand ils s'emparent du pouvoir. Allergique à l'utopie sous toutes ses formes, tu es toujours prêt à croire le pire, s'agissant des membres de ton espèce (Huston, 1995 : 73),

lui écrit par voie posthume Nancy Huston. Pour croire au collectif, il faut croire à l'identité et Gary ne croit guère à la sienne. On se souvient de son fameux « Je me suis toujours été un autre" (Gary, 1981: 30). Mais il évoque souvent dans ses romans la conclusion de Les Mots et les choses de Foucault: "l'homme (...) est une invention récente " (Foucault, 1966 : 398), même s'il pense, par ailleurs, qu'il faudra sans doute encore quelque millier d'années avant que l'Homme n'apparaisse vraiment. Or, ce qu'il aperçoit surtout dans «la prétendue révolution française de 1968 [ce sont] des fils à papa (...) bien nourris, bien habillés, bien éduqués et bien installés » (Gary, $2005: 189$ ) dans un monde de souffrance, de misère et de malnutrition: "On a dit que les émeutiers n'avaient aucun dessein. C'est assez juste: ils ne faisaient que vomir le monde » (Gary, $2005: 189)$ et son état de chiennerie permanent.

\section{La volonté générale contre la volonté du général !}

5 Mais, pour beaucoup, Mai 68, ce n'est pas la chiennerie mais la chienlit. Pour l'ancien Résistant du bataillon Lorraine, il ne saurait s'agir de trahir le général de Gaulle, l'incarnation et le symbole de la France libre. Mais celui auquel Gary est resté irréductiblement fidèle, c'est bien moins l'homme politique que celui qui incarne la France, la grandeur de son histoire et de sa résistance, la transcendance de son destin, un homme d'exception que Paul Audy nomme à très juste titre, en reprenant le terme d'Henri Corbin, une "incarnation "imaginale" " (Gary, 2000: 126). Bien sûr, l'absence d'une véritable cohérence programmatique (Michéa, 2008: 126) ${ }^{3}$ d'un mouvement surtout dirigé contre le Vieux, tout cela heurte son attachement profond à l'esprit gaulliste. Mais l'on ne sait pas précisément ce qu'il en est des opinions politiques de celui qui écrivait en 1958: "J'ai pour principe de couper immédiatement tout rapport avec les personnalités qui deviennent trop éminentes politiquement.» (Gary, 1958) Le général de Gaulle est un symbole pluriel : pour Gary, il est le héraut d'une conception éthique, plus encore, mystique de la France ; pour les soixante-huitards, étudiants ou ouvriers, il incarne l'obstacle à ce que Gary pourra, dans un de ses articles, réduire à des revendications de « salaires, de prix, d'emplois, de logements, de voitures, d'impôts, de congés » (Gary, 2000: 66), sans ignorer cependant qu'il en va plus profondément d'un combat contre «le conservatisme moral ambiant " (Savatier, 2008) d'une époque en pleine mutation. Or, si l'esprit soixante-huitard semble bien loin de l'attitude très 
ambiguë de Gary à l'égard de l'idéalisme en général, de l'individualisme ou de l'utopisme, il n'en demeure pas moins que Gary est trop sensible à toutes les injustices sociales ou raciales pour ne pas devoir écrire «que, d'une certaine façon, inattendue, les étudiants de Paris sont en train de renouer, en lui donnant cette fois un contenu authentique, avec la vénérable tradition humanitaire française et même avec notre "mission universelle" » (Savatier, 2008). Lui-même n'appartient-il pas ouvertement à la longue tradition "de ces objecteurs de conscience et de ces avocats du diable qui, depuis Shakespeare et Montaigne, n'ont cessé d'accompagner le cheminement de la modernité européenne » (Larat, $2014: 135)$ ?

Deux romans, Chien blanc (1970) et Gros-Câlin (1974), évoquent subrepticement Mai 68, et quelques autres la figure du général de Gaulle. Mais tous mettent en scène ou réfléchissent les problèmes qu'affronte la civilisation occidentale moderne face aux solutions radicales du Stalinisme, du Maoïsme, du Castrisme ou des dictatures africaines ou sud-américaines que Gary n'a eu de cesse de critiquer, de dénoncer, de honnir. Gros-Câlin est signé Émile Ajar, mais Ajar comme Gary, s'ils ne croient pas en l'individu, n'en défendent pas moins, d'abord et contre tout, ses droits imprescriptibles, quelles que soient sa race, sa classe, sa nationalité, son sexe! Mais tous deux savent aussi pertinemment que rien n'est plus dangereux que l'idéal, surtout dans les mains de ceux « qui croient détenir le monopole de la vérité » (Gary, 2005:21) et que tout ce que l'on peut atteindre dans la vie c'est «un monde “à peu près". À peu près juste, à peu près humain... Toute recherche de perfection est totalitaire. » (Gary, $2005: 338$ ) Certes, « en tirant parfois sur le bout du lacet tous les nœuds se défont comme ça d'un seul coup crrac ! comme en mai 68 » (Gary-Ajar, 1974b : 37), avoue Michel Cousin dans GrosCâlin. Mais d'ici à descendre manifester dans la rue... : « la rue, c'est superficiel, dehors, à l'extérieur. Ils font dans les rues. Il faut creuser en profondeur, de l'intérieur " (GaryAjar, 2012 : 49). Gary raconte dans Chien blanc cette anecdote devenue fameuse. On a quand même fini par le convaincre de participer à une manifestation pro-gaulliste, organisée "par les grognards et les gros-bras du gaullisme» (Le Gendre, $2015: 138$ ). Bien que lucide sur les répétitions bégayantes de l'Histoire, le gaullien Gary se prend à croire que «l'Histoire se répète, que le danger menace à nouveau » (Gary, $2000: 112)$ :

un ami me téléphone pour m'annoncer qu'un dernier « carré » de Français libres va descendre cet après-midi les Champs-Élysées. Le dernier "carré », c'est quelque chose à quoi je n'ai jamais pu résister. J'ai horreur des majorités. Elles deviennent toujours menaçantes. On imagine donc mon désarroi lorsque, me présentant plein d'espoir sur les Champs-Élysées, je vois déferler des centaines de milliers d'hommes qui donnent une telle impression d'unanimité que j'en ai la chair de poule. Immédiatement, je me sens contre. Venu pour brandir le drapeau tricolore à la croix de Lorraine sous les risées en compagnie de quelques centaines d'autres irréguliers, je me sens volé. Je leur tourne le dos. Tous les déferlements démographiques, qu'ils soient de gauche ou de droite, me sont odieux. (Gary, $1970: 205$ )

Et de conclure : "Je suis un minoritaire-né », un marginal qui rappellera d'ailleurs en 1979 que «depuis 1956, date de la parution de [Les Racines du ciel], la notion de "marginalité" a conquis toute une jeunesse... Tout le mouvement écologique, notamment, défend cette "marge" de vie, qu'il veut de plus en plus grande. » (Gary, $2005: 349)$

8 Or, cette position aurait pu le rapprocher de certains mouvements libertaires de 68 s'il n'y avait profondément inscrit en lui la prescience de ce qu'analysera Michel Clouscard, vingt ans plus tard, lorsqu'il écrira : 
Le néo-fascisme sera l'ultime expression du libéralisme social libertaire, de l'ensemble qui commence en mai 1968. Sa spécificité tient dans cette formule : tout est permis, mais rien n'est possible. À la permissivité de l'abondance, de la croissance, des nouveaux modèles de consommation, succède l'interdit de la crise, de la pénurie, de la paupérisation absolue. Ces deux composantes historiques fusionnent dans les têtes, dans les esprits, créant ainsi les conditions subjectives du néo-fascisme. (Clouscard, 2014 : 5-6)

Ce néo-fascisme, Gary le repère dans l'aliénation de plus en plus patente à l'irréalité : « télévision, divertissement ou culture, le transfert de l'intérêt vers l'au-delà du réel est, dès maintenant, apparent dans les pays à technologies développées. » (Gary, 2005 : 237) Nous nous acheminons vers une société de l'oubli : la surconsommation, la drogue, la sexualité en sont quelques-unes des formes. Ce que Gary nomme la société de provocation se manifeste par le «triomphe du prêt-à-porter » (Gary, $2005: 265)$ mental, la complaisance pornographique, l'indifférence des masses, la domination de l'«impératif catégorique: "Je veux encore plus de tout, et surtout de moi-même" " (Gary, 2005 : 267). Il n'y a aucune issue, autre que pathologique, à cette provocation continue : «Que ce soit dans les sociétés petit-marxistes ou para-capitalistes, l'évasion dans la dimension de l'irréel est inéluctable. » (Gary, 2005 : 237)

\section{Nous sommes tous des juifs allemands (Gare de Lyon)}

10 Avant d'être désamorcé et récupéré par les organisations politiques, Mai 68 a d'abord été une explosion, une jouissance libertaire du "je", la joie de pouvoir exprimer des positions singulières, une révolte plurielle, des désirs polymorphes, une libération d'imagination et d'affects : «Il était impossible de découvrir pour quoi les étudiants se battaient, en termes de changements politiquement constructifs. Ils se contentaient de réagir au harcèlement quotidien de notre civilisation » (Gary, $2005: 198)$. Contre tous les pouvoirs implacables - «pouvoir nucléaire, économique, militaire, industriel, mécanisé, organisé, anonyme, impudent » (Gary, 2005 : 198) - la jeunesse réagit d'une façon peut-être d'autant plus péremptoire dans ses slogans qu'elle éprouve cruellement ce que Gary avait déjà mis en lumière à propos de l'Amérique dans Adieu Gary Cooper :

J'annonçais donc dans ce roman l'Amérique d'aujourd'hui, l'Amérique du doute, de l'anxiété, de l'angoisse, de la vulnérabilité [...]. D'après la réaction des jeunes aujourd'hui encore, parce que je reçois pas mal de lettres au sujet de ce roman, c'est un livre qui a beaucoup marqué les jeunes. C'était écrit juste avant Mai 68 et il y avait déjà là des éléments qui annonçaient Mai 68 dans mes personnages. (Gary, 1969 : 87-88)

11 Parmi ces éléments, il en est un qui chapeaute en définitive tous les autres : la bonne conscience occidentale que la suite des traumatismes $d u x^{e}$ a fini par effondrer, d'abord en Europe, ensuite aux USA, avant qu'elle ne redresse la tête vers un modèle de jouissance égoïste, matérielle, étendu à toute la planète. Ce modèle que Dany-Robert Dufour dénonce, ce «nouvel espace sociétal prosaïque, trivial et nihiliste où la valeur, désormais unifiée dans le système de la marchandise, peut passer d'une main à l'autre. (...) En un sens, l'adjectif "libéral" désigne la condition d'un homme "libéré" de toute attache à [ses] anciennes valeurs symboliques" (Dufour, 2011: 19). Autrement dit, l'homme libéré de la Valeur, de ces «nobles mensonges » (Gary, $2005: 141)$ n'est autre que l'homme aliéné, l'homme-barbaque dont les résultats sont "le vandalisme, l'alcoolisme, la mécanisation du sexe, la toxicomanie et les émeutes incessantes 
(...) conséquences normales de la démystification réaliste, (...) une régression. » (Gary, 2005 : 141) Ainsi,

ce que je voulais dire par Adieu Gary Cooper, c'était pendant la guerre du Vietnam, c'était adieu l'Amérique sûre d'elle-même, adieu au blanc et au noir, au sens valeur, au sens traitre, au sens positif, adieu au personnage que jouait Gary Cooper à l'écran, au personnage qu'incarnait Gary Cooper à l'écran, c'est-à-dire adieu à l'Amérique tranquille, sûre de ses valeurs, sûre de son droit et sûre de toujours gagner à la fin. (Gary, 2014a: 87)

Le roman, écrit avant 68 mais paru en 1969, fait un sort à bien des illusions. Passons rapidement sur la critique de la religion: «Il y avait des églises dans tous les coins, mais autant aller prendre un café-crème, c'est tout aussi réconfortant » (Gary, 1969 : 134).

13 L'inanité de la révolte et la position paradoxale des jeunes bourgeois sont des cibles également faciles à atteindre :

La révolte des jeunes bourgeois contre la bourgeoisie était condamnée au canular ou au fascisme, la seule différence entre les deux étant quelques millions de morts. Il y a quelque chose de profondément répugnant à voir un cortège d'étudiants venir les bras ouverts vers des ouvriers en grève, comme des femelles en chaleur devant les vrais mâles. (Gary, $1969: 135)$

Pourtant, en "vrai mâle", Gary ne s'est jamais fait faute d'accueillir la "femelle"... Cet inconfort spirituel et physique qui naît du hiatus entre le rêve et la réalité a été bel et bien un des ressorts de Mai 68. Sur le mode contrasté du style ajarien (Chaudier, 2014), Gary fait dire à Cousin, le personnage de Gros-Câlin, en visite chez l'une de ses "bonnes putes":

C'est toujours merveilleux lorsque toutes les barrières tombent et on se retrouve tous ensemble, on se rejoint. Au moment de la grande peur, en mai 68 - je n'ai pas osé sortir de chez moi pendant trois semaines à cause de l'espoir, de la fin de l'impossible, [...] - j'ai vu une fois, en regardant prudemment par la fenêtre, des gens qui se rencontraient au milieu de la rue et qui se parlaient. (Gary, 1974b : 72)

Pendant quelques semaines, un rêve d'humanité, de rencontre, d'amour a pu s'incarner dans une sorte de bonheur ponctuel collectif urbain devenu une valeur en soi, tout comme le malheur collectif peut aussi, provisoirement, réunir les hommes. C'est ainsi qu'on pouvait lire, sur les murs de la Sorbonne : «Je prends mes désirs pour la réalité, car je crois en la réalité » ou "Ceux qui prennent leurs désirs pour des réalités sont ceux qui croient à la réalité de leurs désirs" (Besançon, 2007 : 159). Au-delà des aspirations personnelles, les désirs qui trouvent leur expression formelle dans une idéologie sont, pour Gary, à l'image des "plus beaux gâteaux [...] ce sont ceux qui n'existent pas. Dieu. Le communisme. La fraternité. L'Homme, avec un H grand comme ça. » (Gary, 1969: 67) Or, quand on veut installer l'Homme majuscule sur terre, cela donne les camps d'extermination, le Goulag, Hiroshima. Est-ce à dire que plutôt qu'adapter la réalité à ses désirs, il convienne de faire l'inverse ? Certainement pas, car « un homme qui peut s'adapter à la réalité n'est qu'un enfant de pute » (Gary, 1969 : 95)! De ce point de vue, le Baron, personnage énigmatique qui passe d'œuvre en œuvre chez Gary, ne risque plus rien des effets délétères d'une telle compromission : «Il est à la poursuite de l'Homme majuscule et finit, dans son exigence, par refuser tout contact avec l'humain.» (Gary, 2005 : 345). Ayant coupé tous les ponts - sauf ceux de la parodie et de la dérision - avec la réalité empirique - hormis celle de l'alcool ingurgité -, il a, par la même occasion, coupé tous les liens qui pouvaient encore le relier à autrui, au 
désir, au mouvement, à la vie : «Le Baron est en lutte constante par la parodie contre ses propres illusions lyriques. » (Gary, 1974a : 313)

Pourtant, aux yeux de Gary, les étudiants ont raison - comme avaient sans doute aussi raison dans leur indignation les "étudiants révoltés de 1848 » (Gary, 1972: 201) -, même s'il ne peut les suivre dans leurs illusions : «Les jeunes Français me semblent toujours des jeunes bourgeois, souvent des jeunes bourgeois révoltés, mais leur révolte rappelle celle des générations précédentes.» (Gary, 2014b:15) Parmi les raisons qui justifient un tel jugement de valeur, il n'est pas impossible que ces étudiants manifestent cet excès de "je", d'individualisme, de singularité, auquel Romain Gary a toujours tenté d'échapper :

Mon je ne me suffit pas comme vie, et c'est ce qui fait de moi un romancier, j'écris des romans pour aller chez les autres. Si mon je m'est souvent insupportable, ce n'est pas à cause de mes limitations et infirmités personnelles, mais à cause de celles du je humain en général. On est toujours piégé dans un je. (Gary, 1974a : 156)

Les jeunes Français bourgeois, même dans leur révolte, ressemblent trop à eux-mêmes. Ils revendiquent la possibilité d'exprimer en toute liberté les aspirations de leur moi alors que pour Gary, la vraie liberté et la seule conquête consisteraient à parvenir à « l'étrangeté radicale, la capacité d'être totalement autre " (Braunstein et Pépin, 2014 : 56). Conquête et liberté pourtant paradoxales puisqu'elles finissent par se confondre à la perte, l'aliénation et la schizophrénie dans Europa que Nancy Huston définit à juste titre comme

un tour de force formel qui laisse le lecteur pantois, chancelant: toutes les ficelles de l'art romanesque sont visibles, toutes sont tirées, et elles finissent par nous ligoter complètement l'imaginaire, jusqu'à ce qu'on ne sache plus où donner de la tête, que croire, qui écouter. Chaque personnage invente et rêve les autres personnages; aucune scène n'a statut de réalité première; toutes les existences se reflètent ou se rejouent mutuellement. (Huston, $1995: 76)^{4}$

18 Enfin, ce sont des jeunes bourgeois cultivés qui fréquentent l'université et manifestent. Or, certes,

la culture est une Puissance favorable, mais une Puissance tout de même. (...) La différence entre les Allemands héritiers d'une "immense" culture et les Simbas incultes, c'est que les Simbas mangeaient leurs victimes, tandis que les Allemands les transformaient en savon. Ce besoin de propreté, c'est la culture. (Gary, 1965 : 530)

19 Et il ne pouvait échapper à Gary que « [l]orsque les étudiants criaient "À bas la culturealibi !" ils obéissaient fidèlement à la culture, c'était la culture qui parlait. (Gary, 1972 : 159) Pourtant, même s'ils n'utilisent peut-être pas le bon medium et ne visent pas la bonne finalité, les étudiants ont raison de tenter de recoudre, ne serait-ce que pour quelques jours, ne serait-ce que dans l'inversion carnavalesque, ces entités irréconciliables, culture et société, utopie et réalité, idéal et existence. Et sans doute ont-ils d'autant plus raison aux yeux de Gary lorsqu'il le font en recourant à la mascarade, à l'ironie, à l'humour, à l'inversion, à l'antiphrase et au chiasme, en jouant avec la langue, en s'attaquant à la langue, autrement dit au fondement de la culture. Lorsqu'en décembre 1791, on porta Mozart à la fosse commune, sans le savoir,

l'aristocratie et la bourgeoisie assistèrent là à leurs propres obsèques et à celles de l'Europe... Ce fut fini ce jour-là. (...) L'Europe est morte du privilège culturel. Le dédoublement de sa personnalité - la culture d'un côté, la vie des hommes de l'autre -cette schizophrénie, ne pouvait manquer de finir dans des crises meurtrières... (Gary, 1972 : 202) 
Europa est certainement le roman qui met en scène, de la façon la plus hallucinée, le discours de Gary sur cette schizophrénie qui, selon lui, a mené au fascisme, tout comme elle nous mène, depuis plusieurs décennies, dans l'impasse de la société de provocation. Et pourtant, les étudiants auront toujours raison car leur révolte est une «tentative poétique (...) pour inventer un mythe de l'homme, une mythologie des valeurs, et pour essayer de vivre ce mythe ou du moins s'en rapprocher » (Gary, 1974a : 271). Car ce qui relie sans doute le plus Romain Gary à l'espoir poétique de Mai 68, c'est l'idée sans cesse assenée chez lui que "l'homme sans mythologie de l'homme, c'est de la barbaque [et que] étant donné le néant, il n'y a plus aucune raison de se gêner » (Gary, 1974a : 271) et l'on peut alors sans souci envisager aussi bien le cannibalisme comme solution à la crise de la faim dans le monde. Mais s'il est dangereux de vivre sans mythologie, il l'est tout autant de vouloir concrétiser cet absolu. Si l'on veut homogénéiser ou dialectiser la pensée de Gary, lui appliquer une cohérence globale, on se heurte très vite à une circularité infernale et stérilisante. La seule échappatoire au hiatus entre culture et réalité demeure cependant l'imagination, la fiction, le roman dont Gary a défendu la cause dans Pour Sganarelle. Car ce n'est qu'en puisant dans la fiction que l'on peut espérer trouver « la foi exaltante et la force nécessaire pour sortir de la fiction. » (Gary, $1972: 107)$

\section{Soyez réalistes demandez l'impossible (Censier)}

21 Il est probable que Romain Gary a intuitivement pressenti que la révolte de 68, en entraînant bien des bouleversements dans l'existence quotidienne, ait participé paradoxalement à la formation d'un monde

dangereusement rapproché, ridiculement réduit à nos proportions, instrumentalisé à l'excès, un monde pour ainsi dire mis à portée de la main [...] Peu à peu la lassitude nous a gagnés, notre vitalité s'est muée en tristesse [...] nous nous sentons en général si prisonniers de la «Puissance » du réel et des vicissitudes qu'elle nous impose, que nous ne rêvons plus notre avenir. (Gary, 2000 : 120-121)

Ce sont ces formes du rêve guidées par "une recherche de valeurs permanentes, incontestables, (...) un "idéal" de vie digne d'être défendu » (Gary, $2005: 285)$ que Gary a toujours poursuivies dans ses romans où "un certain excès d'aspiration et d'humanisme idéaliste fait à chaque page sa propre autocritique et même sa propre parodie " (Gary, $2005: 287)$. Il y a dans ses romans quelque chose du «Soyez réalistes demandez l'impossible " que les étudiants écrivaient sur les murs de Censier. Cet impossible réaliste doit d'abord aller au-delà des trois impasses historiques du $\mathrm{xx}^{\mathrm{e}}$ siècle :

Le fascisme a fait disparaître l'individu dans les foules fanatiques. Le communisme a interdit à l'individu de parler tout en le collectivisant dans des masses hurlant les mêmes slogans. L'ultralibralisme a réduit l'individu à son fonctionnement pulsionnel, préalable à sa grégarisation dans de vastes troupeaux de consommateurs. (Dufour, 2011 : 331)

Et Dany-Robert Dufour de conclure que

l'individu n'a encore jamais existé (...) Il faut donc construire un nouvel individualisme altruiste, c'est-à-dire un individualisme enfin sympathique [contre] les dérives de l'individualisme égoïste qui, lui, est pathologique, [sans retomber dans] la benoîte morale de l'amour du prochain prônée par le grand récit monothéiste. (Dufour, $2011: 332$ ) 
La seule morale d'amour universel qui puisse faire naître un jour l'homme à lui-même et aux autres, Gary l'a placée dans

La féminité... Elle seule est civilisation: ce que les femmes ont défendu instinctivement à travers les âges et exactement tout ce que les hommes ont gâché ou détruit. C'est sur les valeurs féminines que tout se joue, se perd ou se gagne. Faut-il rappeler que la voix du Christ était une voix essentiellement féminine? Compassion, tendresse, amour... (Gary, 2005 : 346)

Gary retrouve ainsi «ce qu'Orwell appelait la common decency, c'est-à-dire ces vertus humaines élémentaires que sont, par exemple, la loyauté, l'honnêteté, la bienveillance ou la générosité. » (Michéa, 2008 : 24) Pourtant si la Valeur, féminine et maternelle, est le fondement de toute vie digne d'être vécue, "puisque l'être humain ne peut pas vivre de lui-même" (Gary, 2005: 348), encore faut-il avoir le courage de la mettre "à l'épreuve de ces acides que sont l'humour, l'ironie, la bouffonnerie et même un certain cynisme (...). Ce cynisme-là est d'abord un stoïcisme... » (Gary, 2005 : 286). Aussi bien, Gary ne manque pas de moduler: "Mon "mysticisme" de l'amour, même de l'amour physique, n'est fonction que de ma vie personnelle. Je n'entends pas en faire un prêche [ni un] absolu. Je propose un "à peu près".» (Gary, 2005 : 348) La nuance était sans doute d'autant plus nécessaire que Gary n'est pas sans connaître sa réputation de "coureur". Lesley Blanch ne manquera pas de souligner que

[1]e grand séducteur, ou l'image virile, conquérante, qu'il a toujours cultivée, si irritante pour les autres hommes, si attirante pour les femmes, ces personnages cédaient parfois la place à un autre. C'était celui de l'auteur, amant romantique, plein de tendresse pour l'idéal de la féminité fragile. Mais on pourrait décrire cette attitude comme une façon de penser toute théorique, et qui, brièvement, triomphait d'appétits plus élémentaires. (Blanch, 1998 : 135)

\section{C'est pas fini ! (Boulevard Saint Michel)}

«On a tiré une fausse morale de la fable : la vraie, c'est que les cigales chantent toujours. Elles font en somme aux fourmis une réponse pleine de courage et de fierté : elles continuent à chanter » (Gary, 1979 :139).

Il y a eu des fourmis soixante-huitardes - celles des partis politiques, des syndicats, des obédiences gauchistes - et puis, il y a eu aussi les cigales de Mai 68, jeunes gens plus proches du Peace and love et des moutons du Larzac que de l'embrigadement. C'est pour celles-ci que Gary a sans doute pu éprouver par moments le sentiment d'une certaine fraternité de pensée. Mais à cinquante-quatre ans, Romain Gary ne pouvait que se tenir à distance des mots d'ordre et de désordre. Il appartient finalement davantage, en tant qu'homme, à une tradition du juste milieu, de l'à-peu-près, et laisse le soin à ses personnages romanesques de vivre les excès idéalistes dont il voit, à travers ce que subit sa femme, tous les dangers et les désillusions à venir. Et puis, enfin, la grande révolte contre le mauvais Père, il n'a cessé de la vivre dans sa lutte permanente contre tous les discours et régimes autoritaires. Il s'était choisi depuis trop longtemps dans la figure du Général de Gaulle, l'homme qui incarnait pour lui le bon Père, dans l'image de la France, le pays qui représentait le rêve maternel, pour participer à leur déstabilisation, même si lui aussi a sans doute rêvé bien souvent de retrouver « sous les pavés, la plage ». 


\section{BIBLIOGRAPHIE}

ANISSIMov, Myriam (2006). Romain Gary, le caméléon. Paris : Denoël.

BESANÇON, Julien (2007). Journal mural Mai 68. Paris : Tchou éditeur.

BLANCH, Lesley (1998). Romain, un regard particulier (Jean Lambert trad.). Arles, Actes Sud.

BonA, Dominique (1987). Romain Gary. Paris : Mercure de France.

BRAUNSTEIN, Florence et PÉPIN, Jean-François (2014). « L'existoir. Brève biographie imaginaire », in Jean-François Hangouët et Paul Audi (orgs.), L'Herne : Romain Gary. Paris : Les Cahiers de l'Herne, pp. 54-59.

CHAUDIER, Stéphane (2014). « "Dieu ait son cul”. Le style de Momo-Rosa », in Romain Gary, revue Europe, $\mathrm{n}^{\circ}$ 1022-1023, pp. 145-162.

Dufour, Dany-Robert (2011). L'individu qui vient... après le libéralisme. Paris : Denoël.

FoucAult, Michel (1966). Les Mots et les choses. Paris : Gallimard.

GARY, Romain (1958). « Lettre à Henri Hoppenot », in Kerwin Spire (2014), « Romain Gary, franctireur du gaullisme?» [consulté le 16 août 2018] <URL : http://journals.openedition.org/ litteratures/285>.

GARY, Romain (1965). Pour Sganarelle. Paris: Gallimard.

GARY, Romain Gary (1969). Adieu Gary Cooper. Paris: Gallimard.

GARY, Romain (1970). Chien blanc. Paris : Gallimard.

GARY, Romain (1972). Europa. Paris: Gallimard.

GARY, Romain (1974a). La Nuit sera calme. Paris : Gallimard.

GARY, Romain (Émile Ajar) (1974b). Gros-Câlin. Paris : Mercure de France.

GARY, Romain (1979). Les Clowns lyriques. Paris : Gallimard.

GARY, Romain (1981). Vie et mort d'Émile Ajar. Paris : Gallimard.

GARY, Romain (2000). Ode à l'homme qui fut la France. Paris : Gallimard, postface de Paul Audi.

GARY, Romain (2005). L'Affaire homme. Paris : Gallimard.

GARY, Romain (2014a). Le Sens de ma vie, entretien. Paris : Gallimard.

GARY, Romain (2014b), « Un picaro moderne », entretien avec K. A. Jelenski, in Jean-François Hangouët et Paul Audi (orgs.). L'Herne : Romain Gary. Paris : Les Cahiers de l'Herne, pp. 11-15. GUÉGAN, Gérard, NARODETZKI, Jean-Franklin (2008). Mai 68 à l'usage des moins de vingt ans. Paris : Actes Sud.

Huston, Nancy (1995). Tombeau de Romain Gary. Arles : Actes Sud.

LARAT, Fabrice (2014). « Portrait de Romain Gary en intellectuel engagé », in Jean-François Hangouët et Paul Audi (orgs.). Romain Gary. Paris : Cahiers de l'Herne, pp. 129-135.

LE GENDRE, Bertrand (2015). De Gaulle et Mauriac. Paris : Fayard.

MichÉA, Jean-Claude (2008). La Double pensée. Paris : Flammarion. 
SAVATIER, Thierry (2008). « Romain Gary et mai 68 » [on-line] in Les mauvaises fréquentations. Le blog de Thierry Savatier. <URL : http://savatier.blog.lemonde.fr/>

Todorov, Tzvetan (2002). « Romain Gary, géographie de la mémoire », in Mireille Sacotte (org.),

Romain Gary et la pluralité des mondes. Paris : Puf, pp. 99-110.

\section{NOTES}

1. «En France, la commission de censure interdit Les Oiseaux vont mourir au Pérou, par neuf voix contre dix, car "traitant en termes tragiques et sans espoir de la frigidité féminine". L'interdiction ne sera levée que par une intervention de George Gorse. Aux États-Unis, [...] les Oiseaux... est le premier film à mériter la catégorie X. » (Bona, 1987 : 282)

2. À cet égard, parmi les multiples interprétations et mésinterprétations de ce "mouvement » ( ?), nous adhérons plutôt à celle qui souligne qu'il se fit d'abord contre la majorité des étudiants et que subversion et insurrection se sont étendues « à une portion considérable de l'espace social sans avoir eu à passer par un endoctrinement et une idéologie préalablement partagée.» (Guégan et Narodetzki, $1998: 24$ )

3. «Ce printemps magnifique était (...) celui de la contradiction généralisée, chaque forme de contestation à peine apparue suscitant à son tour une contestation symétrique.» (Michéa, 2008 : La double pensée, 126)

4. Il est amusant de penser que, dans un registre d'écriture formelle très différent, Claude Simon publiera un an plus tard Triptyque où là non plus " aucune scène n'a statut de réalité première ". Mais Europa diffuse une réflexion sur la culture et le réel de façon plus corrosive sans doute par l' inquiétante étrangeté de la dépersonnalisation de ses personnages.

\section{RÉSUMÉS}

Romain Gary ne participe pas vraiment à Mai 68, mais toute son œuvre interroge les problèmes qui ont secoué cette époque: la sexualité, la drogue, la consommation, l'écologie, les totalitarismes... Sa position politique est plutôt de gauche. Mais elle est rendue compliquée non seulement par ses relations avec Jean Seberg ou le général De Gaulle, mais aussi par ses doutes identitaires, sa conception de l'humain, sa critique de l'idéalisme et du matérialisme et sa propre position de romancier. Cette complexité explique peut-être que bien des pages de ses romans et entretiens apparaissent aujourd'hui visionnaires.

Romain Gary is not really involved in May 68, but all his work questions the troubles that shook this period: sexuality, drug, consumption, ecology, totalitarisms... He rather have left-wing political opinions. But these are complicated by his relationships with Jean Seberg or the general De Gaulle, also by his doubts about identity, his human conception, his criticism of idealism or materialism et his novelist's position. Perhaps this complexity explains that many pages of his novels or interviews seem to be nowadays visionary ones. 
INDEX

Keywords : Ethics, idealism, materialism, human/inhuman, identity, novel

Mots-clés : ethique, idéalisme, matérialisme, humain/inhumain, identité, roman

\section{AUTEUR}

JACQUES ISOLERY

Université de Corse - UMR CNRS 6240 LISA

isolery[at]univ-corse.fr 